\title{
The Dimensional Representation and the Metric Structure of Similarity Data ${ }^{1}$
}

\author{
AMOS 'TVERSKY \\ Hebrew University of Jerusalem, Jerusalem, Israel \\ AND \\ David H. Krantz \\ University of Michigan, Ann Arbor, Michigan 48104
}

\begin{abstract}
A set of ordinal assumptions, formulated in terms of a given multidimensional stimulus set, is shown to yield essentially unique additive difference measurement of dissimilarity, or psychological distance. According to this model, dissimilarity judgments between multidimensional objects are regarded as composed of two independent processes: an intradimensional subtractive process, and an interdimensional additive process. Although the additive difference measurement model generalizes traditional metric models, the conditions under which it satisfies the metric axioms impose severe restrictions on the measurement scales. The implications of the results for the representation of similarity data by metric and/or dimensional models are discussed.
\end{abstract}

\section{INTRODUCTION}

In a recent review, Beals, Krantz, and Tversky (1968) presentcd and discusscd axiomatizations of a number of the fundamental geometric models of multidimensional scaling, including metrics with additive segments, additive difference metrics, and a special case of both, the (Minkowski) power metrics. The exposition relied on a paper by Beals and Krantz (1967) and on several unpublished results. The main purpose of this paper is to publish proofs of the theorems previously discussed. In particular, Theorems 2 and 3 of Beals et al (1968) are included in Theorems 1 and 2 of the present paper. The statements about the power metric in the previous paper are consequences of a much-improved characterization presented here. We show that the

1 This research was supported by NSF Grants GB 4947 and GB 6782 to the University of Michigan. Preparation of the manuscript was assisted by PHS Grant GM 1231 to the University of Michigan. 
only additive difference metrics with additive segments are the power metrics (eliminating a major axiom, A7, used previously to characterize the power metric).

In addition, we analyze a more general model than any previously considered in multidimensional scaling, called decomposability (Eq. 2 below). This model is of interest in its own right and serves also to unify our treatment of the different facets of the additive difference model, interdimensional additivity and intradimensional subtractivity. We also take this opportunity to discuss the concept of "psychological dimension" implicit in the present approach.

The models to be discussed are all generalizations of the power metric (Minkowski $r$-metric, $L^{p}$ metric), according to which the distance between the points $x=\left(x_{1}, \ldots, x_{n}\right)$ and $y=\left(y_{1}, \ldots, y_{n}\right)$ is given by

$$
d(x, y)=\left[\sum_{i=1}^{n}\left|x_{i}-y_{i}\right|^{r}\right]^{1 / r}, \quad r \geqslant 1 .
$$

The well-known Euclidean and city-block metrics are special cases of the power metric, where $r$ equals 2 or 1 , respectively. Equation 1 incorporates three fundamental assumptions.

(a) Decomposability. The distance between points is a function of componentwise contributions.

(b) Intradimensional Subtractivity. Each component-wise contribution is the absolute value of the scale difference.

(c) Interdimensional Additivity. The distance is a function of the sum of component-wise contributions.

In psychological applications, stimuli $x=\left(x_{1}, \ldots, x_{n}\right)$ and $y=\left(y_{1}, \ldots, y_{n}\right)$ may be characterized by their components, yet the numerical values of the corresponding courdinates in the psychological space are not known in advance. Furthermore, the actual distances between such points are also unknown; instead, one has some measure of dissimilarity, or "psychological distance," that is monotonically related to metric distance. Regarding the power metric as a model of "psychological distance," it can be generalized in three steps, with two branches.

The most general equation embodies only decomposability (a):

$$
d(x, y)=F\left[\phi_{1}\left(x_{1}, y_{1}\right), \ldots, \phi_{n}\left(x_{n}, y_{n}\right)\right],
$$

where $F$ is an increasing function ${ }^{2}$ in each of its $n$ arguments, and each $\phi_{i}$ is a symmetric

${ }^{2}$ Throughout the paper, we shall use the terms increasing and decreasing to mean strictly monotonically increasing and decreasing functions, respectively. All functions are real-valued. 
function of two (nominal scale) arguments, $x_{i}, y_{i}$, with $\phi_{i}\left(x_{i}, x_{i}\right)<\phi_{i}\left(x_{i}, y_{i}\right)$ if $x_{i} \neq y_{i}$.

If subtractivity (b) is assumed we can write Eq. 2 as

$$
d(x, y)=F\left(\left|X_{1}-Y_{1}\right|, \ldots,\left|X_{n}-Y_{n}\right|\right)
$$

where we have replaced $\phi_{i}\left(x_{i}, y_{i}\right)$ by $\left|X_{i}-Y_{i}\right|$ and $X_{i}=f_{i}\left(x_{i}\right)$ is the $i$-th coordinate of stimulus $x$ in the psychological space. Note that $X_{i}$ is a real number, though $x_{i}$ need not be.

If additivity (c) alone is assumed, the power metric is generalized to

$$
d(x, y)=F\left[\sum_{i=1}^{n} \phi_{i}\left(x_{i}, y_{i}\right)\right]
$$

where $F$ is now an increasing function in one argument. Note that subtractivity is an intradimensional property as it refers to differences along the same dimension while additivity is an interdimensional property as it refers to summation across different dimensions. Equations 3 and 4 are both special cases of Eq. 2, but represcnt separate branches in generalization.

If both additivity and subtractivity are assumed we obtain the additive difference model defined by

$$
d(x, y)=F\left[\sum_{i=1}^{n} \phi_{i}\left(\left|X_{i}-Y_{i}\right|\right)\right]
$$

where $F$ and $\phi_{i}, i=1, \ldots, n$, are all increasing functions of one variable. Equation 5 is a special case of both Eqs. 3 and 4 (for the $\phi_{i}$ would be redundant in Eq. 3, since $F$ is already a general function of $n$ variables, increasing in each variable). The power metric is, clearly, a special case of the additive difference model, where all $\phi_{i}$ are the same convex power function and $F$ is its inverse.

The purpose of the present paper is the analysis of the models represented by Eqs. 1-5 from the viewpoint of measurement theory. As in multidimensional psychophysics, we assume that the stimuli can be labeled by a vector of (nominal scale) values on $n$ dimensions, and that an ordinal measure of dissimilarity between the stimuli is given. The conditions (or axioms) which are necessary and/or sufficient to establish decomposability (2), subtractivity (3), additivity (4), or both (5) are presented in the next section. The conditions under which the additive difference model (5) satisfies the metric axioms, and an axiomatic ordinal characterization of the power metric (1) are discussed in Sec. II. The final section explores the implications of the results to the analysis of similarity. 


\section{Additive and Subtractive Dimensions}

The theory is formulated in terms of a product set $A=A_{1} \times \cdots \times A_{n}$ and a real-valued function $M$ defined on $A \times A$. For example, $A$ may be a set of color patches described in terms of their brightness, hue, and saturation, or a set of facial expressions described in terms of their intensity and pleasure, or a set of rectangles described by their height and width. The function $M$ assigns to every pair of elements $x, y$ in $A$, a scale value $M(x, y)$, which is an ordinal measure of the dissimilarity, or the "psychological distance" between them. We use the letters $u, v, w, x, y, z$ (with or without primes) to denote elements of $A$, with $x=\left(x_{1}, \ldots, x_{n}\right)$ where $x_{i}$ denotes the $i$-th component of $x$.

Several comments about the primitives are in order. First, the factorial representation of a given set of stimuli need not be unique. Rectangles, for example, may be characterized in terms of height and width or in terms of area and shape. The present theory, therefore, depends on a particular factorial characterization of the stimuli. Second, the components of the stimuli may be nominal scale values and they need not be real numbers. Third, in referring to the number of factors of $A$, denoted $n$, we discard inessential factors that have only a single value. Fourth, the use of an ordinal scale $M$ is not essential; it simplifies the axioms, but it can be replaced by an abstract order relation on $A \times A$, provided that a separability axiom (Debreu, 1954) is introduced.

In the case of color patches, we expect that the psychological dimensions, brightness, hue, and saturation, are more likely to satisfy the axioms than the more arbitrary coordinates, luminance, dominant wavelength, and purity. In the case of rectangles, the axioms may be better satisfied for height $\times$ width than for area $\times$ shape representations, or vice versa, depending on how the rectangles are perceived; this could vary between subjects or be influenced by context.

To further simplify the statement of the axioms, a notion of betweenness is defined. We say that $y$ is between $x$ and $z$, denoted $x|y| z$, if the following two conditions hold

(i) $M(x, z) \geqslant M(x, y), M(y, z)$

(ii) $x_{i}=y_{i}=z_{i}$ for any $i$ where $x_{i}=z_{i}$.

Thus, $y$ is between $x$ and $z$ if it coincides with $x$ and $z$ on any dimensions on which they are equal, and if its dissimilarity from each of them does not exceed $M(x, z)$. The basic axioms may now be stated as follows.

(Al) If $x \neq y$, then $M(x, x)=M(y, y)<M(y, x)=M(x, y)$.

(A2) If $M(w, x) \leqslant \alpha \leqslant M(w, z)$, then there exists $y$ such that $x|y| z$ and $M(w, y)=\alpha$. 
(A3) For any $i=1, \ldots, n$, if $x_{i}=x_{i}{ }^{\prime}, y_{i}=y_{i}{ }^{\prime}, z_{i}=z_{i}{ }^{\prime}, w_{i}=w_{i}{ }^{\prime}$, and for all $j \neq i, x_{j}=z_{j}, x_{j}^{\prime}=z_{j}^{\prime}, y_{j}=w_{j}$, and $y_{j}^{\prime}=w_{j}^{\prime}$, then $M(x, y) \leqslant M\left(x^{\prime}, y^{\prime}\right)$ if and only if $M(z, w) \leqslant M\left(z^{\prime}, w^{\prime}\right)$.

(A4) For any $i=1, \ldots, n$, if $x_{i}=z_{i}, x_{i}{ }^{\prime}=z_{i}{ }^{\prime}, y_{i}=w_{i}, y_{i}{ }^{\prime}=w_{i}{ }^{\prime}$, and for all $j \neq i, x_{j}=x_{j}{ }^{\prime}, y_{j}=y_{j}{ }^{\prime}, z_{j}=z_{j}{ }^{\prime}$, and $w_{j}=w_{j}{ }^{\prime}$, then $M(x, y) \leqslant M\left(x^{\prime}, y^{\prime}\right)$ if and only if $M(z, w) \leqslant M\left(z^{\prime}, w^{\prime}\right)$.

(A5) For any $i=1, \ldots, n$, if for all $j \neq i, x_{j}=y_{j}=z_{j}=w_{j}$, and $x|y| z$, then

(i) if $y|z| w$ and $y \neq z$, then $x|y| w$ and $x|z| w$

(ii) if $x|z| w$, then $x|y|$ w and $y|z| w$.

(A6) For any $i=1, \ldots, n$, if for all $j \neq i, x_{j}=y_{j}=z_{j}=x_{j}{ }^{\prime}=y_{j}{ }^{\prime}=z_{j}{ }^{\prime}$, $x|y| z, x^{\prime}\left|y^{\prime}\right| z^{\prime}$, and $M(y, z)=M\left(y^{\prime}, z^{\prime}\right)$, then $M(x, y) \leqslant M\left(x^{\prime}, y^{\prime}\right)$ if and only if $M(x, z) \leqslant M\left(x^{\prime}, z^{\prime}\right)$.

The axioms are practically identical to those discussed in Beals et al. (1968) and we shall not repeat the discussion here, except to remind the reader of a few salient mathematical points. The first axiom asserts that $M$ is minimal between a point and itself, and symmetric in its two arguments. The second axiom is a solvability condition analogous to ones used in other areas of measurement theory (e.g., Luce, 1966). The third axiom is the important independence condition, which is the basic axiom of the general theory of additive conjoint measurement (see Debreu, 1960). It implies (A4); therefore, the latter is assumed only where (A3) is not employed. For the two-dimensional case, however, (A3) is replaced by the following cancellation axiom

$\left(\mathrm{A} 3^{\prime}\right)$

$$
\begin{aligned}
\text { If } & M\left[\left(x_{1}, x_{2}{ }^{\prime}\right),\left(y_{1}, y_{2}{ }^{\prime}\right)\right] \leqslant M\left[\left(x_{1}{ }^{\prime}, x_{2}^{\prime \prime}\right),\left(y_{1}^{\prime}, y_{2}^{\prime \prime}\right)\right] \\
\text { and } & M\left[\left(x_{1}^{\prime}, x_{2}\right),\left(y_{1}^{\prime}, y_{2}\right)\right] \leqslant M\left[\left(x_{1}^{\prime \prime}, x_{2}^{\prime}\right),\left(y_{1}^{\prime \prime}, y_{2}^{\prime}\right)\right], \\
\text { then } & M\left[\left(x_{1}, x_{2}\right),\left(y_{1}, y_{2}\right)\right] \leqslant M\left[\left(x_{1}^{\prime \prime}, x_{2}^{\prime \prime}\right),\left(y_{1}^{\prime \prime}, y_{2}^{\prime \prime}\right)\right] .
\end{aligned}
$$

For an empirical test of (A3) in a three-dimensional case, using schematic faces as stimuli, see Tversky and Krantz (1969).

Finally, both (A5) and (A6) are unidimensional properties that apply to elements of $A$ that coincide on all but one dimension. The statement of (A6) differs slightly from that of Beals et al. (1968), but in the context of (A1), (A2), and (A5), the two are logically equivalent. The major results that are based on the above axioms are summarized in the following theorem. 
Theorem 1. Given a stimulus set satisfying (A1) and (A2) with respect to some $n$-dimensional representation, then the following assertions are valid.

(a) Decomposability is satisfied if and only if (A4) holds; the $\phi_{i}$ are then ordinal scales.

(b) Intradimensional subtractivity is satisfied if and only if (A4), (A5), and (A6) hold. Thus, under these assumptions there exist one-to-one functions $f_{i}$, defined on $A_{i}$, $i=1, \ldots, n$, and an increasing function $F$ such that $M(x, y)=F\left(\left|X_{1}-Y_{1}\right|, \ldots,\left|X_{n}-Y_{n}\right|\right)$, $X_{i}=f_{i}\left(x_{i}\right)$ for all $i$, where each $f_{i}$ is an interval scale.

(c) Interdimensional additivity is satisfied (for $n \geqslant 3$ ) if and only if (A3) holds. Thus, under (A1), (A2), and (A3), there exist functions $\phi_{i}$, defined on $A_{i} \times A_{i}, i=1, \ldots, n$, and an increasing function $F$ such that $M(x, y)=F\left[\sum_{i=1}^{n} \phi_{i}\left(x_{i}, y_{i}\right)\right]$ where all the $\phi_{i}$ are interval scales, with a common unit.

(d) Subtractivity and additivity are satisfied (for $n \geqslant 3$ ) if and only if (A3), (A5), and (A6) hold. Thus, under the above assumptions, there exist functions $f_{i}$, defined on $A_{i}$, $i=1, \ldots, n$, increasing functions $\phi_{i}, i=1, \ldots, n$, and an increasing function $F$ such that $M(x, y)=F\left[\sum_{i=1}^{n} \phi_{i}\left(\left|X_{i}-Y_{i}\right|\right)\right], X_{i}=f_{i}\left(x_{i}\right)$ for all $i$, where the $f_{i}$ are interval scales and the $\phi_{i}$ are interval scales with a common unit.

(e) If $n=2$, assertions (c) and (d) are still valid, provided (A3) is replaced by (A3').

The rest of this section is devoted to the proof of Theorem 1 .

To prove part (a) of Theorem 1, we define the function $\phi_{i}$ by holding all components constant except the $i$-th and setting $\phi_{i}-M$. More precisely, let $u$ be some fixed element of $A$. Let $U_{i}\left(x_{i}\right)$ be the element of $A$ whose $j$-th component equals $u_{j}$ for $j \neq i$ and whose $i$-th component is $x_{i}$. Define

$$
\phi_{i}\left(x_{i}, y_{i}\right)=M\left[U_{i}\left(x_{i}\right), U_{i}\left(y_{i}\right)\right] .
$$

From (A1) and (A2), $\phi_{i}$ is a symmetric function, with $\phi_{i}\left(x_{i}, x_{i}\right)<\phi_{i}\left(x_{i}, y_{i}\right)$ if $x_{i} \neq y_{i}$.

Let $T_{i}$ be the range of the function $\phi_{i}$. We define a function $F$ of $n$ real variables, on $T_{1} \cdots \cdots T_{n}$, by

$$
F\left[\phi_{1}\left(x_{1}, y_{1}\right), \ldots, \phi_{n}\left(x_{n}, y_{n}\right)\right]=M(x, y) .
$$

We now use (A4) to prove that $F$ is well-defined and increasing in each variable; this establishes decomposability.

Suppose that $\phi_{i}\left(x_{i}, y_{i}\right)=\phi_{i}\left(x_{i}{ }^{\prime}, y_{i}{ }^{\prime}\right), i=1, \ldots, n$. To show that $F$ is well-defined, we must show that $M(x, y)=M\left(x^{\prime}, y^{\prime}\right)$. For $r=0, \ldots, n$, let $x^{(r)}$ be the element of $A$ whose $i$-th component is $x_{i}$ for $i>r$ and $x_{i}{ }^{\prime}$ for $i \leqslant r$; define $y^{(r)}$ similarly. Then for $i=1, \ldots, n$, we have, for $j \neq i$,

$$
\begin{aligned}
x_{j}^{(i 1)} & =x_{j}^{(i)}, \\
y_{j}^{(i-1)} & =y_{j}^{(i)}, \\
U_{i}\left(x_{i}\right)_{j} & =U_{i}\left(x_{i}{ }^{\prime}\right)_{j}, \\
U_{i}\left(y_{i}\right)_{j} & =U_{i}\left(y_{i}{ }^{\prime}\right)_{j},
\end{aligned}
$$


and for $j=i$,

$$
\begin{aligned}
x_{i}^{(i-1)} & =U_{i}\left(x_{i}\right)_{i}, \\
x_{i}^{(i)} & =U_{i}\left(x_{i}{ }^{\prime}\right)_{i}, \\
y_{i}^{(i-1)} & =U_{i}\left(y_{i}\right)_{i}, \\
y_{i}^{(i)} & =U_{i}\left(y_{i}{ }^{\prime}\right)_{i} .
\end{aligned}
$$

We may thus apply (A4), with $x^{(i-1)}, y^{(i-1)}, x^{(i)}, y^{(i)}$ playing the roles of $x, y, x^{\prime}, y^{\prime}$, and with $U_{i}\left(x_{i}\right), U_{i}\left(y_{i}\right), U_{i}\left(x_{i}{ }^{\prime}\right), U_{i}\left(y_{i}{ }^{\prime}\right)$ playing the roles of $z, w, z^{\prime}, w^{\prime}$, to obtain

$$
M\left[x^{(i-1)}, y^{(i-1)}\right] \geqslant M\left[x^{(i)}, y^{(i)}\right]
$$

if and only if

$$
M\left[U_{i}\left(x_{i}\right), U_{i}\left(y_{i}\right)\right] \geqslant M\left[U_{i}\left(x_{i}{ }^{\prime}\right), U_{i}\left(y_{i}{ }^{\prime}\right)\right]
$$

But by definition of $\phi_{i}$, we have $M\left[U_{i}\left(x_{i}\right), U_{i}\left(y_{i}\right)\right]=M\left[U_{i}\left(x_{i}{ }^{\prime}\right), U_{i}\left(y_{i}{ }^{\prime}\right)\right]$; hence, for $i=1, \ldots, n$, we have $M\left[x^{(i-1)}, y^{(i-1)}\right]=M\left[x^{(i)}, y^{(i)}\right]$. Since $x^{(0)}=x, y^{(0)}=y, x^{(n)}=x^{\prime}$, $y^{(n)}=y^{\prime}$, we have $M(x, y)=M\left(x^{\prime}, y^{\prime}\right)$ as required.

The proof that $F$ is strictly increasing in each variable is exactly parallel to the above, except that one of the equalities $\phi_{i}\left(x_{i}, y_{i}\right)=\phi_{i}\left(x_{i}{ }^{\prime}, y_{i}{ }^{\prime}\right)$ is replaced by strict inequality, and we obtain correspondingly a strict inequality between $M\left[x^{(i-1)}, y^{(i-1)}\right]$ and $M\left[x^{(i)}, y^{(i)}\right]$, hence, between $M(x, y)$ and $M\left(x^{\prime}, y^{\prime}\right)$, as required.

To complete the proof of part (a), we must show that decomposability implies (A4) and that the $\phi_{i}$ are ordinal scales. Suppose that there exist functions $\psi_{i}$ on $A_{i} \times A_{i}$ and $G$ on Range $\psi_{1} \times \cdots \times$ Range $\psi_{n}$, such that $M(x, y)=G\left[\psi_{1}\left(x_{1}, y_{1}\right), \ldots, \psi_{n}\left(x_{n}, y_{n}\right)\right]$. By definition,

$$
\phi_{i}\left(x_{i}, y_{i}\right)=M\left[U_{i}\left(x_{i}\right), U_{i}\left(y_{i}\right)\right]=G\left[\psi_{1}\left(u_{1}, u_{1}\right), \ldots, \psi_{i}\left(x_{i}, y_{i}\right), \ldots, \psi_{n}\left(u_{n}, u_{n}\right)\right],
$$

so $\phi_{i}$ is an increasing function of $\psi_{i}$. Conversely, any increasing functions of the $\phi_{i}$ can be used in a representation of form of Eq. 2, so the $\phi_{i}$ are ordinal scales. To show (A4), it suffices to note that under the hypotheses of (A4), $M(x, y) \geqslant M\left(x^{\prime}, y^{\prime}\right)$ if and only if $\phi_{i}\left(x_{i}, y_{i}\right) \geqslant \phi_{i}\left(x_{i}{ }^{\prime}, y_{i}{ }^{\prime}\right)$ (since the arguments of $F$ are equal for $j \neq i$ ). Similarly, $M(z, w) \geqslant M\left(z^{\prime}, w^{\prime}\right)$ if and only if $\phi_{i}\left(z_{i}, w_{i}\right) \geqslant \phi_{i}\left(z_{i}{ }^{\prime}, w_{i}{ }^{\prime}\right)$. Since $x_{i}=z_{i}$, etc., the conclusion of (A4) follows. This completes part (a).

To prove part (b), we proceed as follows. For each $i$, we must construct $f_{i}$ on $A_{i}$ such that $\phi_{i}\left(x_{i}, y_{i}\right)=h_{i}\left[\left|f_{i}\left(x_{i}\right)-f_{i}\left(y_{i}\right)\right|\right]$, where $\phi_{i}$ is the function constructed above for decomposability, and $h_{i}$ is an increasing function. Intradimensional subtractivity holds if and only if this is possible. This is essentially a one-dimensional problem, 
i.e., the functions $\phi_{j}, j \neq i$, have nothing whatever to do with the possibility of writing $\phi_{i}$ in the needed form. Hence, we identify each $x$ with $U_{i}\left(x_{i}\right)$, holding all coordinates $j \neq i$ fixed at $u_{j}$, and we drop the $i$ subscript for the remainder of the proof. The antecedent conditions of (A5) and (A6), asserting $x_{j}=y_{j}=z_{j}=w_{j}$, or $x_{j}=y_{j}=z_{j}=x_{j}{ }^{\prime}=y_{j}{ }^{\prime}=z_{j}{ }^{\prime}$, for all $j \neq i$, will always be satisfied automatically, since all such coordinates are equal to $u_{j}$. Thus, we apply the one-dimensional axioms (A5) and (A6) without reference to any subscripts.

How can the psychological scale, $f$, be constructed ? Suppose we set $f=0$ for some one element (for concreteness, $f(u)=0$ ), and choose some element $v \neq u$ to have $f(v)=1$. Suppose that $u|v| w$. Suppose also that we construct a sequence $z^{(0)}$, $z^{(1)}, \ldots, z^{(p)}, \ldots, z^{(q)}$ such that $z^{(0)}=u, z^{(p)}=v$, and $z^{(q)}=w$, such that the $\mathfrak{z}^{(j)}$ are equally spaced and ordered - that is, $z^{(j-1)}\left|z^{(j)}\right| z^{(j+1)}$ holds for $j=1, \ldots, q-1$ and $M\left[z^{(j)}, z^{(j+1)}\right]$ is constant, independent of $j$. Intuitively, the $u, v$ interval is composed of $p$ equal intervals; therefore each has psychological length $(1 / p)|f(v)-f(u)|=1 / p$; and $u, w$ is composed of $q$ of the same intervals, thus it has psychological length $q / p$. Hence, we must define $f(w)=q / p$. This indicates the actual construction of the function $f$. This construction is just like dividing a meter stick (e.g., $u, v$ ) into $p=1000$ equal parts, and then seeing how many millimeters (e.g., $q$ ) are required to span $u, w$; The length of $u, w$ in meters is then $q / 1000$. Equality of millimetric divisions is replaced by equisimilarity, $M\left[z^{(j-1)}, z^{(j)}\right]=M\left[z^{(j)}, z^{(j+1)}\right]=\cdots$; ordering of the millimeters along a straight line corresponds to the requirement $z^{(j-1)}\left|z^{(j)}\right| z^{(j+1)}$, which is inferred from proximity data via the above definition of betweenness. It remains to be proved that this equisection process will really give consistent results (e.g., suppose the $z^{(j)}$ sequence is replaced by one with smaller or larger steps) and that the function $f$, so constructed, will predict the order of all proximity comparisons. The roles of (A5) and (A6) in such a proof are quite clear. Axiom (A5), if satisfied, guarantees that the "betweenness" inferred from proximity ordering is well-behaved; for example, if $z^{(j-1)}\left|z^{(j)}\right| z^{(j+1)}$, and $z^{(j)}\left|z^{(j+1)}\right| z^{(j+2)}$, we know also that $z^{(j-1)}\left|z^{(j+1)}\right| z^{(j+2)}$. Axiom (A6) guarantees that two equal steps $y, z$ and $y^{\prime}, z^{\prime}$, in different parts of the scale, add on in the same way. For example, we can deduce, for the above sequence $z^{(j)}$, that $M\left[z^{(j)}, z^{(j+2)}\right]$ is also constant, independent of $j$, using (A6).

We have taken pains to make clear the intuitive method of scale construction, and the reasons why properties (A5) and (A6) are needed to guarantee that it will work, because the formal proof does not proceed by showing in detail the consequences of the construction of $f$ just sketched. We could so proceed, but it would be very redundant to do so. The similarity of the above constructive scheme to measurement of physical length is more than a superficial analogy-the measurement-theoretic analysis of extensive measurement is directly applicable. What we do is reduce this case directly to one of the standard theorems on extensive measurement (Krantz, 1968), and use the real-valued function which that theorem tells us exists to construct $f$. 
The counting-of-equal-units process is contained in the proof of the extensive measurement theorem and so does not appear explicitly here. ${ }^{3}$

Lct $T$ denote the interior of the range of $\phi$ (remember, subscripts are dropped). By (A1) and (A2), $T$ is an open interval of real numbers, with lower bound $\phi(x, x)$; it may or may not be bounded above. We define a binary operation, $(s, t) \rightarrow s * t$, on a subset of $T \times T$, as follows:

$$
\begin{aligned}
\text { If } & s=\phi(x, y), \quad t=\phi(y, z), \quad \text { and } \quad x|y| z, \\
\text { then } & s * t=\phi(x, z) .
\end{aligned}
$$

That $*$ is well-defined follows readily from (A6). Denote by $B$ the subset of $T \times T$ for which $*$ is defined.

Lemma. The quadruple $\left\langle T, B,{ }^{*}, \geqslant\right\rangle$ (where $\geqslant$ is the natural ordering of real numbers) is an Archimedean positive ordered local semigroup in the sense of Krantz (1968); that is:

$1 . \geqslant$ is a total order;

2. if $(s, t) \in B, s \geqslant s^{\prime}, t \geqslant t^{\prime}$, then $\left(t^{\prime}, s^{\prime}\right) \in B$;

3. if $(r, s),(r * s, t) \in B$, then $(s, t),(r, s * t) \in B$ and $(r * s) * t=r *(s * t)$;

4. if $r \geqslant s$ and $(r, t) \in B$, then $r * t \geqslant s * t$ and $t * r \geqslant t * s$;

5. if $(s, t) \in B$, then $s * t>s$;

6. if $t>r$, then there exists $s \in T$ with $(r, s) \in B$ and $t \geqslant r * s$;

7. for any $s, t$ in $T$, the set of integers $m$ such that $m s$ is defined and $t \geqslant m s$ is finite [where $1 s$ is defined as $s$, and if $(m-1) s$ is defined, and $((m-1) s, s) \in B$, then $m s$ is defined as $(m-1) s * s]$.

\section{Proof.}

1. The first property is trivial.

2. Assume $s=\phi(x, y), t=\phi(y, z), x|y| z$. Since $s^{\prime} \leqslant s$, by (A2) there exists $y^{\prime}$ with $x\left|y^{\prime}\right| y$ and $\phi\left(x, y^{\prime}\right)=s^{\prime}$. By (A5), $y^{\prime}|y| z$, so $\phi\left(y^{\prime}, z\right) \geqslant t \geqslant t^{\prime}$. By (A2), there exists $z^{\prime}$ with $y^{\prime}\left|z^{\prime}\right| z$ and $\phi\left(y^{\prime}, z^{\prime}\right)=t^{\prime}$. Since (A5) implies $z^{\prime} \mid y^{\prime} ; x$, and $\phi\left(z^{\prime}, y^{\prime}\right)=t^{\prime}, \phi\left(y^{\prime}, x\right)=s^{\prime}$, we have $\left(t^{\prime}, s^{\prime}\right) \in B$.

3. Using (A2) and (A6) we can find $x, y, z$, and $w$ such that $r=\phi(x, y)$, $s=\phi(y, z), t=\phi(z, w)$, with $x|y| z$ and $x|z| w$. By (A5), $y|z| w$, so $(s, t) \in B$, and $s * t=\phi(y, w)$. By (A5), $x|y| w$, so $(r, s * t) \in B$ and $r *(s * t)=\phi(x, w)=$ $(r * s) * t$.

\section{Follows immediately from (A6).}

${ }^{3}$ It can also be shown that the present assumptions allow us to construct a system satisfying the axioms of Suppes and Winet (1955), who gave the first axiomatization of intradimensional subtractivity. The major advance in our treatment, besides simplicity of assumptions, is the elimination of their primitive (undefined) ordering along the dimension. 
5. Let $s=\phi(x, y), t=\phi(y, z)$ where $x|y| z$. Since $T$ is the interior of the range of $\phi, t>\phi(y, y)=$ glb $T$; thus, $y \neq z$. We can apply (A6) to the triples $x, y, z$ and $x, y, y$ to deduce that $\phi(x, z)>\phi(x, y)$, or $s * t>s$.

6. Follows immediately from (A2).

7. Assume the contrary, i.e., that there exist $s, t$ such that $m s$ is defined for every positive integer $m$ and $t \geqslant m s$ for all $m$. Let $t=\phi(x, z)$ and $s=\phi\left(x, x^{(1)}\right)$, where $x\left|x^{(1)}\right| z$. Since $s * s$ is defined and $\leqslant t$, we know from (A6) that $\phi\left(x^{(1)}, z\right) \geqslant s$; thus, we can find $x^{(2)}$ with $x^{(1)}\left|x^{(2)}\right| z$ and $\phi\left(x^{(1)}, x^{(2)}\right)-s$. This process can be continued indefinitely, obtaining $x^{(0)}=x, x^{(1)}, x^{(2)}, \ldots$, such that $x^{(i)}\left|x^{(i+1)}\right| x^{(i+2)}$ and $x^{(i)}\left|x^{(i+1)}\right| z$ for $i=0,1,2, \ldots$. We have $m s=\phi\left(x, x^{(m)}\right) \leqslant t$ for all $m$. The sequence $\phi\left(x, x^{(m)}\right)$ is increasing and bounded above, so it has a limit, $r$, in $T$. Thus, for some $y$, $x|y| z$ and $\phi(x, y)=r$.

Now choose $y^{\prime}$ with $x\left|y^{\prime}\right| y$ and with $\phi\left(y^{\prime}, y\right)=s$. By property $5, \phi(x, y)=$ $\phi\left(x, y^{\prime}\right) * \phi\left(y^{\prime}, y\right)>\phi\left(x, y^{\prime}\right)$. Therefore there exists some $m$ such that $\phi(x, y) \geqslant$ $\phi\left(x, x^{(m)}\right)>\phi\left(x, y^{\prime}\right)$. Since $\phi\left(x, x^{(m)}\right)>\phi\left(x, y^{\prime}\right)$ and $\phi\left(x^{(m)}, x^{(m+1)}\right)=s=\phi\left(y^{\prime}, y\right)$, it follows from (A6) that $\phi\left(x, x^{(m+1)}\right)>\phi(x, y)$, a contradiction.

Q.E.D.

By Theorem 3.1 of Krantz (1968), there exists an increasing real-valued function $g$ on $T$, unique up to multiplication by a positive constant, such that $g(s * t)=g(s)+g(t)$.

Now define $f$ as follows. Choose fixed $u, v$ with $u \neq v$. Let $f(u)=0$. If $x \neq u$, define

$$
f(x)=\left\{\begin{array}{ll}
g(\phi(u, x)) & \text { if } u|x| v \\
-g(\phi(u, x)) & \text { if } x|u| v .
\end{array} \text { or } \quad u|v| x\right.
$$

(If there is an upper bound of form $\phi\left(u^{\prime}, v^{\prime}\right)$ for $T$, then choosing $u$ distinct from $u^{\prime}, v^{\prime}$ is sufficient to guarantee that $\phi(u, x) \in T$ for all $x \neq u$.)

It remains to be shown that $\phi(x, y)$ is an increasing function of $|f(x)-f(y)|$. Now $x$ can be in one of three regions, defined by $x|u| v, u|x| v$, and $u|v| x$, and so can $y$; this yields nine cases. The proof in each case is very simple; we carry through two cases for illustration.

(i) Suppose $u|v| x, u|v| y$. Now $x|u| y$ implies (with $u|v| y$, by A5) that $x|u| v$; this with $u|v| x$ means that $\phi(x, u)=\phi(x, v)$, contradicting the fact that $g \phi(x, u)=g \phi(x, v)+g \phi(u, v)$. Hence, without loss of generality, we can assume that $u|x| y$. We then have

as required.

$$
\begin{aligned}
g \phi(x, y) & =g \phi(u, y)-g \phi(u, x) \\
& =f(y)-f(x)
\end{aligned}
$$

(ii) Suppose $x|u| v, u|y| v$. By (A5), we have $x|u| y$, hence,

as required.

$$
\begin{aligned}
g \phi(x, y) & =g \phi(x, u)+g \phi(u, y) \\
& =-f(x)+f(y)
\end{aligned}
$$


Similar treatment of other cases completes the proof that (A5), (A6) imply intradimensional subtractivity [in the presence of (A1) and (A2)] for each dimension. The converse follows immediately from properties of absolute values of differences of real numbers.

Finally, to establish that $f$ is an interval scale, note that for any other $f^{\prime}, h$, with $h$ increasing, such that $\phi(x, y)=h\left[\left|f^{\prime}(x)-f^{\prime}(y)\right|\right], h^{-1}$ is an additive representation of the structure $\langle T, B, *, \geqslant\rangle$, so by the uniqueness theorem for extensive measurement $h^{-1}=\alpha g$, where $\alpha>0$. Therefore

$$
\begin{aligned}
\left|f^{\prime}(x)-f^{\prime}(y)\right| & =\alpha g \phi(x, y) \\
& -\alpha|f(x)-f(y)| .
\end{aligned}
$$

It follows in straightforward fashion that $f$ and $f^{\prime}$ are related by a linear transformation, $f^{\prime}=\beta f+\gamma$, where $|\beta|=\alpha, \gamma=f^{\prime}(u)$. This completes part (b).

To prove part (c) of Theorem 1, we note first that (A4) follows from (A3), so that Eq. 2 (decomposability) applies. It suffices to show that appropriate monotonic transforms of the $\phi_{i}\left(x_{i}, y_{i}\right)$ can be chosen so that the function of $n$ variables, $F$, can be written as a function of the sum of component-wise contributions. This is a standard problem in additive conjoint measurement (see Luce, 1966). We will not review here the construction of the appropriate transformed $\phi_{i}$ 's; it is similar to the constructions of extensive measurement (see Krantz, 1968, and Luce and Tukey, 1964, for details). The simplest proof is obtaincd by reducing the theorem to that of Debreu (1960).

Debreu's theorem gives sufficient conditions for a function $F$ on a product set $T=T_{1} \times \cdots \times T_{n}$ to be written as $F(t)=G\left(\sum_{i=1}^{n} h_{i}\left(t_{i}\right)\right)$. The conditions (for $n \geqslant 3$ ) are:

1. Each $T_{i}$ is a connected and separable topological space.

2. If $r_{j}=t_{j}, j \neq i$, and $F(r) \leqslant \alpha \leqslant F(t)$, then there exists $s$, with $s_{j}=r_{j}=t_{j}$ for $j \neq i$, and $F(s)=\alpha$.

3. If $s_{j}=s_{j}{ }^{\prime}$ and $t_{j}=t_{j}{ }^{\prime}$ for all $j \neq i$, and $s_{i}=t_{i}, s_{i}{ }^{\prime}=t_{i}{ }^{\prime}$, then $F(s) \leqslant F(t)$ if and only if $F\left(s^{\prime}\right) \leqslant F\left(t^{\prime}\right)$.

4. For any $s$, the sets $\{t \mid F(s) \geqslant F(t)\}$ and $\{t \mid F(t) \geqslant F(s)\}$ are closed in the product topology on $T$.

To apply this theorem, let $T_{i}$ be the range of $\phi_{i}$ (for part (b), we had let $T_{i}$ be the interior of the range). Each $T_{i}$ is an interval of real numbers so Condition 1 is satisfied (indeed, Debreu uses Condition 1 only to replace his topological spaces by isomorphic real intervals). Condition 2 follows readily from (A1) and (A2), while Condition 3 is essentially the same as (A3). Only Condition 4 offers a bit of difficulty; it is established as follows.

To show that $\{t \mid F(t) \geqslant F(s)\}$ is closed, let $t$ be an arbitrary element not in this set, i.e., $F(t)<F(s)$. We must show that there is a neighborhood $N$ of $t$, in $T$, such that 
$F\left(t^{\prime}\right)<F(s)$ for all $t^{\prime} \in N$. We define elements $t_{i}{ }^{*} \in T_{i}$ inductively as follows. Suppose that $t_{i}{ }^{*}$ has been defined for $i<k$, where $1 \leqslant k \leqslant n$, and that $F\left(t_{1}{ }^{*}, \ldots, t_{k-1}^{*}, t_{k}, \ldots, t_{n}\right)$ (which we denote $\alpha_{k-1}$ for short) satisfies the inequality $F(t) \leqslant \alpha_{k-1}<F(s)$. Define $t_{k}{ }^{*}$ as follows. If $t_{k}=\operatorname{lub} T_{k}$, let $t_{k}{ }^{*}=t_{k}$. (Note that in this case, $\alpha_{k}=\alpha_{k-1}$ and we are done with this step.) Otherwise, choose $r_{k} \in T_{k}$, with $r_{k}>t_{k}$. Since $F\left(t_{1}{ }^{*}, \ldots, t_{k-1}^{*}, r_{k}\right.$, $\left.t_{k+1}, \ldots, t_{n}\right)>\alpha_{k-1} \geqslant F(t)$, we can choose a number $\alpha_{k}$ satisfying

$$
F(t) \leqslant \alpha_{k-1}<\alpha_{k}<\min \left\{F(s), F\left(t_{\mathbf{1}}^{*}, \ldots, t_{k-1}^{*}, r_{k}, t_{k+1}, \ldots, t_{n}\right)\right\}
$$

and can then choose $t_{k}{ }^{*}$ with $t_{k}<t_{k}{ }^{*}<r_{k}$ such that $F\left(t_{1}{ }^{*}, \ldots, t_{k}{ }^{*}, t_{k+1}, \ldots, t_{n}\right)=\alpha_{k}$ as required. Denoting $\left(t_{1}{ }^{*}, \ldots, t_{n}{ }^{*}\right)$ by $t^{*}$, we have, by construction, $F(t) \leqslant F\left(t^{*}\right)<F(s)$, and for each $i$, either $t_{i}{ }^{*}=\operatorname{lub} T_{i}$ or $t_{i}<t_{i}{ }^{*}$. If $t_{i}{ }^{*}=\operatorname{lub} T_{i}$, let $N_{i}=T_{i}$; otherwise, let $N_{i}=\left\{t_{i}{ }^{\prime} \mid t_{i}{ }^{\prime}<t_{i}{ }^{*}\right\}$. In either case, $N_{i}$ is open in $T_{i}$, so $N=N_{1} \times \cdots \times N_{n}$ is open in $T$. Clearly, $F\left(t^{\prime}\right) \leqslant F\left(t^{*}\right)$ for all $t^{\prime} \in N$, so $N$ is the required neighborhood.

The other half of Condition 4 is proved in the same way, with inequalities reversed.

Applying Debreu's theorem now shows that (A3) implies interdimensional additivity. The converse is straightforward. The uniqueness follows from standard uniqueness results for $n$-dimensional conjoint measurement (e.g., Debreu, 1960; Luce, 1966).

For part (d) of Theorem 1, it suffices to note that under the assumptions (A3), (A5), (A6), (b) and (c) both apply, yielding

$$
\begin{aligned}
M(x, y) & =F\left(\left|f_{1}\left(x_{1}\right)-f_{1}\left(y_{1}\right)\right|, \ldots,\left|f_{n}\left(x_{n}\right)-f_{n}\left(y_{n}\right)\right|\right) \\
& =G\left[\sum_{i=1}^{n} \psi_{i}\left(x_{i}, y_{i}\right)\right] .
\end{aligned}
$$

By the ordinal uniqueness theorem for decomposability, we have $\psi_{i}\left(x_{i}, y_{i}\right)=$ $\phi_{i}\left(\left|f_{i}\left(x_{i}\right)-f_{i}\left(y_{i}\right)\right|\right)$, where $\phi_{i}$ is an increasing function, $i=1, \ldots, n$. Substituting $\phi_{i}$ for $\psi_{i}$ yields the additive difference model (Eq. 5).

The functions $\phi_{i}$ in Eq. 5 can be normalized so that $\phi_{i}(0)=0$; this makes them ratio scales.

Finally, we note that Debreu's theorem applied only for $n \geqslant 3$; for $n=2$, he proved the same result, assuming the Thomson condition from the theory of webs in place of Condition 3. But this latter condition follows from ( $\left.\mathrm{A} 3^{\prime}\right)$ just as his Condition 3 follows from (A3). This establishes part (e) of the theorem. For $n=1$, only part (b) (which was proved without any restriction on $n$ ) is of interest-decomposability and additivity are trivial.

\section{Additive Difference Metrics}

The additive difference model (Eq. 5), established in Theorem 1, provides a measurement of dissimilarity that generalizes the power metric (Eq. 1). Nevertheless, it need 
not be a metric distance function. A metric distance function or a metric, for short, is a function from $A \times A$ into the reals, which assigns to every pair of points $x, y$ a number $d(x, y)$, called their distance, such that the following conditions are satisfied.

(MI) Positivity. $d(x, x)=0$ and $d(x, y)>0$ if $x \neq y$.

(M2) SYMMETRY. $d(x, y)=d(y, x)$.

(M3) The Triangle Inequality. $d(x, y)+d(y, z) \geqslant d(x, z)$.

The metric axioms, by themselves, impose very little structure. They are satisfied, for example, by the trivial form $d(x, x)=0$ and $d(x, y)=1$ for $x \neq y$. In any of the more interesting geometries, (e.g., Euclidean, Riemannian) it is further assumed that any pair of points is joined by a segment along which distances are additive. Stated formally, we introduce

(M4) Segmental Additivity. For any distinct points $x$ and $z$, there exists a set of points $Y$, and a one-to-one mapping, $f$, from $Y$ onto some real interval $[a, b]$, such that $x$, z are in $Y, f(x)=a, f(z)=b$, and for any $u$, $v$ in $Y, d(u, v)=|f(u)-f(v)|$. Hence, in particular, (M4) implies that for all $y$ in $Y, d(x, y)+d(y, z)=d(x, z)$. An ordinal axiomatization of a metric with additive segments (i.e., a scale satisfying (M1), (M2), (M3), and (M4)) has been developed by Beals and Krantz(1967). Note that the above axioms are applicable to any arbitrary set of points. If our object set, however, has a product structure (like the object sets discussed in the previous section) it may be of interest to investigate a weaker version of (M4) where segmental additivity is postulated for points that differ only on one dimension. Stated formally, we obtain

(M4') Segmental Additivity. (M4) holds for any points $x$, $z$ that differ on one dimension only.

We refer to a metric scale as a metric with additive segments or as a proper metric depending on whether it satisfies (M4) or (M4'), respectively. An additive difference model (Eq. 5) is said to be compatible with a metric, if there exists a metric, $d$, satisfying

$$
M(w, x) \leqslant M(y, z) \quad \text { if and only if } \quad d(w, x) \leqslant d(y, z),
$$

for all $w, x, y, z$ in $A$. This section is devoted to the investigation of the constraints imposed on the additive difference model by the assumption that it is compatible with a metric satisfying (M4) or (M4').

From the assumption that an additive difference model is compatible with a proper metric, it follows that there exists some increasing function $F$ such that for all $x, y$ in $A$

$$
d(x, y)-F\left[\sum_{i=1}^{n} \phi_{i}\left(\left|X_{i}-Y_{i}\right|\right)\right]
$$


If $x, z$ differ on the $i$-th dimension only, then any $y$ on the additive segment $Y$ joining them must also differ from them on the $i$-th dimension only (otherwise, it is easy to show that the triangle inequality would be violated). For such a $y$, we have (normalizing so $\left.\phi_{i}(0)=0\right)$ :

$$
F\left[\phi_{i}\left(\left|X_{i}-Y_{i}\right|\right)\right] \mid F\left[\phi_{i}\left(\left|Y_{i} \quad Z_{i}\right|\right)\right]=F\left[\phi_{i}\left(\left|X_{i} \cdot Z_{i}\right|\right)\right] .
$$

Since $\left|X_{i}-Z_{i}\right| \geqslant\left|X_{i}-Y_{i}\right|,\left|Y_{i}-Z_{i}\right|$ (i.e., $x|y| z$ ), we have $\left|X_{i}-Z_{i}\right|=$ $\left|X_{i}-Y_{i}\right|+\left|Y_{i}-Z_{i}\right|$. Putting $\alpha=\left|X_{i}-Y_{i}\right|, \beta=\left|Y_{i}-Z_{i}\right|$ yields

$$
F\left[\phi_{i}(\alpha)\right]+F\left[\phi_{i}(\beta)\right]=F\left[\phi_{i}(\alpha+\beta)\right] .
$$

By defining $\psi_{i}(\alpha)=F\left[\phi_{i}(\alpha)\right]$ we obtain the equation $\psi_{i}(\alpha)+\psi_{i}(\beta)=\psi_{i}(\alpha+\beta)$, for all $\alpha, \beta$ in a nonnegative real interval including 0 . The only monotonic solution is $\psi_{i}(\alpha)=t_{i} \alpha$. Since $F$ is one-to-one, it has an inverse, $F^{-1}$, satisfying

$$
F^{-1}\left[\psi_{i}(\alpha)\right]=F^{-1}\left(t_{i} \alpha\right)=\phi_{i}(\alpha) \quad \text { for } \quad i=1, \ldots, n \text {. }
$$

But since $F^{-1}$ is independent of $i$, all the $\phi_{i}$ 's must be identical up to a change of unit of their domain. Hence, under (M4 $4^{\prime}$ ), there exists some function $\phi$ such that, for any $i$, $\phi_{i}(\alpha)=\phi\left(t_{i} \alpha\right)$.

Next, we study the consequences of the triangle inequality. Consider three points $x$, $y, z$ in $A$ such that $x$ and $y$ differ only on the $i$ th dimension, while $y$ and $z$ differ only on the $j$-th dimension. From (6) and (M3), we obtain

$$
F\left[\phi_{i}\left(\left|X_{i}-Y_{i}\right|\right)\right]+F\left[\phi_{j}\left(\left|Y_{j}-Z_{j}\right|\right)\right] \geqslant F\left[\phi_{i}\left(\left|X_{i}-Y_{i}\right|\right)+\phi_{j}\left(\left|Y_{j}-Z_{j}\right|\right)\right]
$$

Letting $\left|X_{i}-Y_{i}\right|=\alpha$, and $\left|Y_{j}-Z_{j}\right|=\beta$, yields

$$
F\left[\phi_{i}(\alpha)\right]+F\left[\phi_{j}(\beta)\right] \geqslant F\left[\phi_{i}(\alpha)+\phi_{j}(\beta)\right]
$$

But since $F\left[\phi_{i}(\alpha)\right]=t_{i} \alpha, i=1, \ldots, n$, and $\phi_{i}(\alpha)=F^{-1}\left(t_{i} \alpha\right)=\phi\left(t_{i} \alpha\right)$ we can apply $F^{1}=\phi$ to both sides of the above inequality to obtain $\phi\left(F\left[\phi_{i}(\alpha)\right]+F\left[\phi_{j}(\beta)\right]\right)=$ $\phi\left(t_{i} \alpha+t_{j} \beta\right) \geqslant \phi\left(t_{i} \alpha\right)+\phi\left(t_{j} \beta\right)$ and $\phi$ is, therefore, a superadditive function. (Note that convexity implies superadditivity, provided $\phi(0)=0$, but not conversely.) The above discussion has, thus, demonstrated the first part of the following:

ThEorem 2. For an additive difference model to be compatible with a proper metric, it is necessary that the functions $\phi_{i}, i=1, \ldots, n$, satisfy

(i) $\phi_{i}(\alpha)=\phi\left(t_{i} \alpha\right)$

(ii) $\phi(\alpha+\beta) \geqslant \phi(\alpha)+\phi(\beta)$. 
It is sufficient that $\phi$ is of the form

$$
\phi(\alpha)=p\left(e^{q \alpha}-1\right)^{r}, \quad \text { for } p>0, q>0, \quad r \geqslant 1
$$

Thus, according to Theorem 2, any proper metric that is compatible with an additive difference model must be of the form

$$
d(x, y)=\phi^{-1}\left[\sum_{i=1}^{n} \phi\left(t_{i}\left|X_{i}-Y_{i}\right|\right)\right] \quad \text { for some superadditive } \phi
$$

It is readily seen that the power metric provides one example of a proper metric where $\phi(\alpha)=\alpha^{r}$, for some $r \geqslant 1$. Another example of a proper metric, called the exponential metric, is obtained by letting $\phi(\alpha)==e^{u \alpha}-1$, for some $q>0$. An extension of both the power metric and the exponential metric is given in (7). The exponential metric is clearly a special case of (7) where $p=r=1$. The power metric is obtained from (7) by letting $p \rightarrow \infty$ and $q \rightarrow 0$, keeping constant the quantity $k=p q^{r}$. Here, $\phi(\alpha)$ converges uniformly to $k \alpha^{r}$.

To prove the second part of Theorcm 2, we have to show that the form defined by Eqs. 8 and 7, is a proper metric. It is immediately verifiable that (M1), (M2), and (M4') are satisfied, hence we have only to show that (M3) is also satisfied. Letting $\alpha_{i}=t_{i}\left|X_{i}-Y_{i}\right|$, and $\beta_{i}=t_{i}\left|Y_{i}-Z_{i}\right|, i=1, \ldots, n$, the triangle inequality (M3) reduces to

$$
\phi^{-1}\left[\sum_{i=1}^{n} \phi\left(\alpha_{i}\right)\right]+\phi^{-1}\left[\sum_{i=1}^{n} \phi\left(\beta_{i}\right)\right] \geqslant \phi^{-1}\left[\sum_{i=1}^{n} \phi\left(\alpha_{i}+\beta_{i}\right)\right] .
$$

Since $\phi(\alpha)=p\left(e^{q \alpha}-1\right)^{r}$, with $p>0, q>0, r \geqslant 1$, by hypothesis, $\phi^{-1}(\alpha)=$ $(1 / q) \log \left[1+(\alpha / p)^{1 / r}\right]$. Substituting $\phi^{-1}$ in the left-hand side of (9) yields

$$
\begin{aligned}
& \frac{1}{q} \log \left(1+\left[\frac{1}{p} \sum_{i=1}^{n} \phi\left(\alpha_{i}\right)\right]^{1 / r}\right)+\frac{1}{q} \log \left(1+\left[\frac{1}{p} \sum_{i=1}^{n} \phi\left(\beta_{i}\right)\right]^{1 / r}\right) \\
& =\frac{1}{q} \log \left(1+\left[\frac{1}{p} \sum_{i=1}^{n} \phi\left(\alpha_{i}\right)\right]^{1 / r}+\left[\frac{1}{p} \sum_{i=1}^{n} \phi\left(\beta_{i}\right)\right]^{1 / r}+\left[\frac{1}{p^{2}} \sum_{i=1}^{n} \sum_{j=1}^{n} \phi\left(\alpha_{i}\right) \phi\left(\beta_{j}\right)\right]^{1 / r}\right) \\
& \geqslant \frac{1}{q} \log \left(1+\left[\frac{1}{p} \sum_{i=1}^{n} \phi\left(\alpha_{i}\right)\right]^{1 / r}+\left[\frac{1}{p} \sum_{i=1}^{n} \phi\left(\beta_{i}\right)\right]^{1 / r}+\left[\frac{1}{p^{2}} \sum_{i=1}^{n} \phi\left(\alpha_{i}\right) \phi\left(\beta_{i}\right)\right]^{1 / r}\right)
\end{aligned}
$$


(since we have dropped from the last expression inside the parentheses all terms of the form $\phi\left(\alpha_{i}\right) \phi\left(\beta_{j}\right)$ for any $i \neq j$, all of which are nonnegative)

$$
=\frac{1}{q} \log \left[1+\left(\sum_{i=1}^{n} A_{i}^{r}\right)^{1 / r}+\left(\sum_{i=1}^{n} B_{i}^{r}\right)^{1 / r}+\left(\sum_{i=1}^{n}\left[A_{i} B_{i}\right]^{r}\right)^{1 / r}\right]
$$

(by substituting $A_{i}=e^{q \alpha_{i}}-1=\left[(1 / p) \phi\left(\alpha_{i}\right)\right]^{1 / r}$, and $B_{i}=e^{\eta B_{i}}-1=\left[(1 / p) \phi\left(\beta_{i}\right)\right]^{1 / r}$ throughout)

$$
\geqslant \frac{1}{q} \log \left(1+\left[\sum_{i=1}^{n}\left(A_{i}+B_{i}\right)^{r}\right]^{1 / r}+\left[\sum_{i=1}^{n}\left(A_{i} B_{i}\right)^{r}\right]^{1 / r}\right)
$$

(since, by the Minkowksi inequality, for any $r \geqslant 1$,

$$
\begin{aligned}
& \left.\left[\sum_{i=1}^{n} A_{i}^{r}\right]^{1 / r}+\left[\sum_{i=1}^{n} B_{i}^{r}\right]^{1 / r} \geqslant\left[\sum_{i=1}^{n}\left(A_{i}+B_{i}\right)^{r}\right]^{1 / r}\right) \\
& \quad \geqslant \frac{1}{q} \log \left(1+\left[\sum_{i=1}^{n}\left(A_{i} B_{i}+A_{i}+B_{i}\right)^{r}\right]^{1 / r}\right) \text { (again, by the Minkowski inequality) } \\
& =\frac{1}{q} \log \left(1+\left[\sum_{i=1}^{n}\left(e^{q\left(\alpha_{i}+\beta_{i}\right)}-1\right)^{r}\right]^{1 / r}\right) \text { (by substitution) } \\
& =\frac{1}{q} \log \left(1+\left[\frac{1}{p} \sum_{i=1}^{n} p\left(e^{q\left(\alpha_{i}+\beta_{i}\right)}-1\right)^{r}\right]^{1 / r}\right) \\
& =\phi^{-1}\left[\sum_{i=1}^{n} \phi\left(\alpha_{i}+\beta_{i}\right)\right]
\end{aligned}
$$

as required, which completes the proof of Theorem 2 .

Thus, we have seen that (7) and (8) do indeed define proper metrics, but we do not know whether these metrics exhaust the class of additive difference models that are compatible with a proper metric. That is, we do not know whether there exist some other solutions, $\phi$, to Inequality 9.

A close inspection of the last part of the proof of Theorem 2 reveals that the triangle inequality is satisfied with equality only if $\phi\left(\alpha_{i}\right) \phi\left(\beta_{j}\right)=0$ for any $i \neq j$. But since $\phi$ is increasing and $\phi(0)=0$, the above product vanishes only if $\left|X_{i}-Y_{i}\right| \cdot\left|Y_{j}-Z_{j}\right|=0$ for all $i \neq j$. Hence, unless $x, y$, and $z$ differ only on 
one dimension, $y$ must coincide with $x$ and/or $z$. We have, thus, demonstrated the following:

Corollary. The metric defined by (7) and (8) satisfies the triangle equality, i.e., $d(x, y)+d(y, z)=d(x, z)$, for some triple $x, y, z$ if and only if they differ on one dimension only.

Although the power metric can be viewed as a limiting case of the metric defined by (7) and (8), it differs from nonlimiting cases of this family in that it satisfies segmental additivity. In other words, the above corollary does not hold in the limit: for every $x$ and $z$, the triangle equality holds for every $y$ on the affine straight line joining $x$ and $z$. To see this, note that $y$ is on the affine segment joining $x$ and $z$ if there exists $t$, $0 \leqslant t \leqslant 1$, such that $Y_{i}=(1-t) X_{i}+t Z_{i}, i=1, \ldots, n$. We have

$$
\begin{aligned}
d(x, y)-\mid d(y, z)= & {\left[\sum_{i=1}^{n}\left|X_{i} \cdots(1-t) X_{i} \cdots t Z_{i}\right|^{r}\right]^{1 / r} } \\
& +\left[\sum_{i=1}^{n}\left|(1-t) X_{i}+t Z_{i}-Z_{i}\right|^{r}\right]^{1 / r} \\
= & t\left[\sum_{i=1}^{n}\left|X_{i}-Z_{i}\right|^{r}\right]^{1 / \tau}+(1-t)\left[\sum_{i=1}^{n}\left|X_{i}-Z_{i}\right|^{r}\right]^{1 / r} \\
= & d(x, z) .
\end{aligned}
$$

Moreover, the power metric is characterized by this fact: it is the only additive difference metric which is also a metric with additive segments. This is a new result, not announced in Beals et al.(1968). In that paper, an additional axiom(A7) was introduced, to the effect that the affine midpoint of any two points lies on the additive segment between them, and the above result was stated, assuming that (A7) held. However, it turns out to be unnecessary to assume (A7). The precise theorem is as follows:

Theorem 3. Suppose that $M$ satisfies (A1) to (A6) and that $d$ is a metric compatible with $M$, satisfying (M1) to (M4). Then there exist an unique real number $r \geqslant 1$ and real valued functions $X_{i}=f_{i}\left(x_{i}\right)$ on $A_{i}$, such that

$$
d(x, y)=\left[\sum_{i=1}^{n}\left|X_{i}-Y_{i}\right|^{r}\right]^{1 / r}
$$


The proof of Theorem 3 is based on a geometric lemma that appears not to have been known previously. The proof of the lemma was the work of Dr. M. Perles.

Lemma. Let $S$ be an open convex subset of $n$-dimensional Euclidean space. Let $d$ be a metric on $S$, satisfying intradimensional subtractivity with respect to linear coordinates in $S$. If $d$ satisfies segmental additivity (M4), then d is homogeneous, i.e., for any $x, z$ in $S$ and $t$ with $0 \leqslant t \leqslant 1, d[x, x+t(z-x)]=t d(x, z)$. (Another way of expressing homogeneity is that Euclidean lines are additive segments.)

We remark that, as a corollary, for $S$ equal to all of Euclidean $n$-space, the only metrics with additive segments satisfying intradimensional subtractivity are the general Minkowski metrics (see Busemann, 1955, pp. 94-104).

The homogeneity of the metric in Theorem 3 follows from the above lemma, without use of interdimensional additivity. Using homogeneity and interdimensional additivity, we then obtain the power metric from a theorem of Hardy, Littlewood, and Polya (1952) on homogeneous means.

Note that in the above lemma, and in the following proof, the terms "open," "closed," and "continuous" refer exclusively to the natural topology of Euclidean $n$-space, not to the relative topology induced by $S$ nor to the metric topology induced by $d$.

Proof of the Lemma. First consider the case where $t=1 / m$, where $m$ is an integer $\geqslant 1$. Suppose $x, z$ are in $S$. For $k=0,1, \ldots, m$, define $z^{(k)}=x+k(z-x) / m$. By convexity of $S$, each $z^{(k)}$ is in $S$. By intradimensional subtractivity, $d\left[z^{(k-1)}, z^{(k)}\right]=$ $d[x, x+(z-x) / m]$ for $k-1, \ldots, m$. From the triangle inequality, we have

$$
d(x, z) \leqslant \sum_{k=1}^{m} d\left[z^{(k-1)}, z^{(k)}\right]=m d[x, x+(z-x) / m] .
$$

Thus, $d[x, x+(z-x) / m] \geqslant d(x, z) / m$. We need to obtain the opposite inequality to establish the lemma for $t=1 / \mathrm{m}$.

By segmental additivity, we can choose $y^{(0)}, y^{(1)}, \ldots, y^{(m)}$ in $S$ equally spaced on the segment from $x$ to $z$, i.e., $d\left[y^{(i-1)}, y^{(i)}\right]=d(x, z) / m, i=1, \ldots, m$, where $y^{(0)}=x$, $y^{(m)}=z$. Let $x^{(i)}=y^{(i)}-y^{(i-1)}, i=1, \ldots, m$. Since $S$ is open, there is a number $\delta>0$ such that $x+u$ is in $S$ for every $u$ such that $\left|u_{j}\right|<\delta, j=1, \ldots, n$. If we choose $m$ large enough $d(x, z) / m$ is arbitrarily small; since $d$ is monotone in each coordinate, we can obtain $\left|x_{j}^{(i)}\right|=\left|y_{j}^{(i)}-y_{j}^{(i-1)}\right| \leqslant \delta, j=1, \ldots, n$. Thus, $d\left[x, x+x^{(i)}\right]$ is defined and $=d\left[y^{(i-1)}, y^{(i)}\right]=d(x, z) / m$ for $i=1, \ldots, m$. Now $x+(z-x) / m$ is a convex combination of the vectors $x+x^{(i)}$, namely:

$$
\begin{aligned}
\sum_{i=1}^{m}\left[x+x^{(i)}\right] / m & =x+\sum_{i=1}^{m}\left[y^{(i)}-y^{(i-1)}\right] / m \\
& =x+(z-x) / m .
\end{aligned}
$$


Hence, the inequalities $d\left[x, x+x^{(i)}\right] \leqslant d(x, z) / m$ will imply the inequality $d[x, x+(z-x) / m] \leqslant d(x, z) / m$ provided that spheres are convex-if $m$ points are within a given distance of $x$, then any convex combination of them is within that same distance of $x$.

Note that once this convexity result is established, so that $d[x+(z-x) / m]=$ $d(x, z) / m$ for sufficiently large integers $m$, the lemma follows. For by segmental additivity, the same equation holds for $t=k / m$, wherever $0 \leqslant k \leqslant m$ and $m$ is sufficiently large; and by monotonicity, it follows for $0 \leqslant t \leqslant 1$. So we turn to the matter of convexity of spheres. To establish this, we use the Krein-Milman theorem (1940), which asserts that a closed convex set in Euclidean $n$-space is the closed convex hull of the set of its extreme points. (Extreme points are not proper convex combinations of any other points.)

Let $x$ be fixed and let $B(\alpha)$ denote the sphere with center $x$ and radius $\alpha$, i.e., $\{y \mid d(x, y) \leqslant \alpha\}$. Let $C(\alpha)$ be the convex hull of $B(\alpha)$, i.e., the smallest convex set containing $B(\alpha)$, which is the set of all convex combinations of elements of $B(\alpha)$. By continuity of $d$, for $\alpha$ sufficiently small, $B(\alpha)$ is a closed set entirely contained in $S$. The convex hull of a closed set is closed, so $C(\alpha)$ is a closed convex set. By the Krein-Milman theorem, the set of extreme points of $C(\alpha)$, denoted $E(\alpha)$, is the minimal set whose convex hull is dense in $C(\alpha)$. In particular, $B(\alpha)$ contains $E(\alpha)$. Thus, to prove that $B(\alpha)$ is convex (equal to $C(\alpha)$ ), it suffices to show that any convex combination of elements of $E(\alpha)$ is in $B(\alpha)$. It even suffices to show this for convex combinations of form $\sum_{i=1}^{r} y^{(i)} / r$, where $y^{(i)}$ is in $E(\alpha)$, since such combinations are dense in $C(\alpha)$ (any convex combination with rational coefficients can be written in such a form, by letting $r$ be a common denominator).

We showed above, using segmental additivity and intradimensional subtractivity, that $y$ in $B(\alpha)$ implies that $y / r$ is in $C(\alpha / r)$ (there, we used a vector of form $y=z-x$, but this is irrelevant). But if $y$ is in $E(\alpha)$, then $y / r$ must be in $E(\alpha / r)$. For suppose the contrary, i.e., $y / m=\sum_{i=1}^{p} \lambda_{i} w^{(i)}$, where $\lambda_{i} \geqslant 0, \sum_{i=1}^{p} \lambda_{i}=1$, and $w^{(i)}$ is in $C(\alpha / r)$. Since $C(\alpha / r)$ is the set of convex combinations of $B(\alpha / r)$, we can suppose without loss of generality that $w^{(i)}$ is in $B(\alpha / r)$. By the triangle inequality, $r w^{(i)}$ is in $B(\alpha)$; hence $y=\sum_{i=1}^{p} \lambda_{i} r w^{(i)}$ is not in $E(\alpha)$, a contradiction. Hence, a convex combination $\sum_{i=1}^{r} y^{(i)} / r$, with $y^{(i)}$ in $E(\alpha)$, has each $y^{(i)} / r$ in $B(\alpha / r)$. By translation invariance and the triangle inequality, $\sum_{i=1}^{r} y^{(i)} / r$ is in $B(\alpha)$ as required. Thus we have shown that $B(\alpha)$ is convex (for $\alpha$ small enough so that $B(\alpha)$ is a closed set in $S$ ). Since we apply the result only for $\alpha=d(x, z) / m$, for sufficiently largc $m$, wc can assume that $m$ is large enough to obtain $B(\alpha)$ convex. This establishes the lemma completely.

Proof of Theorem 3.

If the conditions of the theorem hold, then $d$ is given by

$$
d(x, y)=\phi^{-1}\left[\sum_{i=1}^{n} \phi\left(\left|X_{i}-Y_{i}\right|\right)\right]
$$


where the constants $t_{i}$ of Theorem 2 are here absorbed into the definition of $X_{i}=f_{i}\left(x_{i}\right)$. Since the metric $d$ is defined on a (convex) cube, the Cartesian product of the ranges of $\left|X_{i}-Y_{i}\right|, i=1, \ldots, n$, the lemma applies. If we let $\alpha_{i}=\left|Z_{i}-X_{i}\right|$, the equation $d[x, x+t(z-x)]=t d(z, x)$ implies the following functional equation.

$$
\phi^{-1}\left[\sum_{i=1}^{n} \phi\left(t \alpha_{i}\right)\right]=t \phi^{-1}\left[\sum_{i=1}^{n} \phi\left(\alpha_{i}\right)\right] .
$$

We shall show that the only solutions to the functional Eq. 10 are of form $\phi(\alpha)=k \alpha^{r}$. To do this, two steps are required. First, we make some remarks about the domains of $\alpha_{i}, t$ for which Eq. 10 has been shown to hold, and show that $\phi$ can be extended to map $[0, \infty)$ onto itself, with (10) valid for $0 \leqslant \alpha_{1}, \ldots, \alpha_{n}, t<\infty$. Second, we show that Eq. 10 implies that $\phi$ satisfies another functional Eq. 12, for which the only solutions are $\phi(\alpha)=k \alpha^{r}$.

By Theorem 2, the additive difference model, $d(x, y)=F\left[\sum_{i=1}^{n} \phi_{i}\left(\left|X_{i}-Y_{i}\right|\right)\right]$, yields a proper metric only if each $\phi_{i}(\alpha)=\phi\left(t_{i} \alpha\right)$, where $\phi=F^{-1}$. Thus, $\phi^{-1}$ is defined for all numbers $\sum_{i=1}^{n} \phi_{i}\left(\left|X_{i}-Y_{i}\right|\right)$, and each $\phi_{i}$ coincides with $\phi$ (except for the factor $t_{i}$ multiplying the argument) in its own domain. Moreover, since the domains of $\phi_{i}$ are intervals, we can choose $\omega>0$ such that for each $i, 1 \leqslant i \leqslant n$, and each $\alpha$, $0 \leqslant \alpha \leqslant \omega / t_{i}, \alpha$ is in the domain of $\phi_{i}$. Note that in particular, if $\omega_{i}=\omega / t_{i}$, $\phi^{-1}\left[\sum_{i=1}^{n} \phi_{i}\left(\omega_{i}\right)\right]=\phi^{-1}[n \phi(\omega)]$ is defined. We denote this quantity by $\Omega$; note that $\phi=F^{-1}$ is defined at least on the interval $[0, \Omega]$. Note finally that for $n=1$, the theorem is immediate, with $r=1(d(x, y)=F[\phi(|X-Y|)]$, but $d$ is additive, so $F \phi=$ multiplication by a constant) (all power metrics are identical for $n=1$ ), while for $n>1, \Omega>\omega$. We can now extend $\phi$ as follows.

For $\alpha \leqslant \omega$, we have by (10)

$$
\begin{aligned}
\phi^{-1}[n \phi(\alpha)] & =\phi^{-1}[n \phi(\omega \alpha / \omega)] \\
& =(\alpha / \omega) \phi^{-1}[n \phi(\omega)] \\
& =\alpha \Omega / \omega .
\end{aligned}
$$

Thus $\phi$ satisfies

$$
\begin{aligned}
\phi(\alpha) & =n \phi(\alpha \omega / \Omega), & & 0 \leqslant \alpha \leqslant \Omega ; \\
\phi^{-1}(a) & =(\Omega / \omega) \phi^{-1}(a / n), & & 0 \leqslant a \leqslant n \phi(\omega) .
\end{aligned}
$$

We can use (11a) to define $\phi$ for $\alpha$ such that the right side of (11a) is defined, i.e., for $0 \leqslant \alpha \leqslant \Omega^{2} / \omega$. Note that $\Omega^{2} / \omega>\Omega$, so this really does extend $\phi$ beyond the interval 
$[0, \Omega]$. Correspondingly, $\phi^{-1}$ satisfies $(1 \mathrm{lb})$ for the extended interval $0 \leqslant a \leqslant n \phi(\Omega)=$ $n^{2} \phi(\omega)$. Moreover $(10)$ is now valid for $0 \leqslant \alpha_{i} \leqslant \Omega$ and $0 \leqslant t \leqslant 1$ :

$$
\begin{aligned}
\phi^{-1}\left[\sum_{i=1}^{n} \phi\left(t \alpha_{i}\right)\right] & =\phi^{-1}\left[\sum_{i=1}^{n} n \phi\left(t \alpha_{i} \omega / \Omega\right)\right] \\
& =\phi^{-1}\left[n \sum_{i=1}^{n} \phi\left(t \alpha_{i} \omega / \Omega\right)\right] \\
& =(\Omega / \omega) \phi^{-1}\left[\sum_{i=1}^{n} \phi\left(t \alpha_{i} \omega / \Omega\right)\right] \\
& =(\Omega / \omega)(t) \phi^{-1}\left[\sum_{i=1}^{n} \phi\left(\alpha_{i} \omega / \Omega\right)\right] \\
& =t \phi^{-1}\left[n \sum_{i=1}^{n} \phi\left(\alpha_{i} \omega / \Omega\right)\right] \\
& =t \phi^{-1}\left[\sum_{i=1}^{n} n \phi\left(\alpha_{i} \omega / \Omega\right)\right] \\
& =t \phi^{-1}\left[\sum_{i=1}^{n} \phi\left(\alpha_{i}\right)\right]
\end{aligned}
$$

(by 10 , applied for $\beta_{i}=\alpha_{i} \omega / \Omega \leqslant \omega$ )

(by 11a).

Repeatedly applying the arguments extends the intervals in which $\phi$ is defined and in which (10) is valid by a factor $\Omega / \omega$ each time. Thus, we can extend $\phi$ to $[0, \infty)$, and have (10) valid for $0 \leqslant \alpha_{i}<\infty, 0 \leqslant t \leqslant 1$. Under these circumstances, it follows immediately that $(10)$ is valid for $0 \leqslant t<\infty$. The function $\phi$, so extended, coincides with the original $\phi$ at least on $[0, \Omega]$.

Now by (11b), we have, for $0 \leqslant \alpha_{i}<\infty$,

$$
\phi^{-1}\left[\sum_{i=1}^{n} \phi\left(\alpha_{i}\right)\right]=(\Omega / \omega) \phi^{-1}\left[\frac{1}{n} \sum_{i=1}^{n} \phi\left(\alpha_{i}\right)\right] .
$$

It follows immediately that the homogeneity equation, (10), applies to the mean-value function, $\phi^{-1}\left[(1 / n) \Sigma \phi\left(\alpha_{i}\right)\right]$ :

$$
\phi^{-1}\left[\frac{1}{n} \sum_{i=1}^{n} \phi\left(t \alpha_{i}\right)\right]=t \phi^{-1}\left[\sum_{i=1}^{n} \phi\left(\alpha_{i}\right)\right] .
$$


By a result in Aczél (1966, p. 153), an increasing function $\varphi$, with $\varphi(0)=0$, satisfying (12) for $0 \leqslant \alpha_{i}<\infty, 0 \leqslant t<\infty$, must be of form $k \alpha^{r}$.

Going back to the original function $\phi$, we see that $\phi(\alpha)=k \alpha^{r}$, at least for $0 \leqslant \alpha \leqslant \Omega$. But now, for any $\left|X_{i}-Y_{i}\right|, i=1, \ldots, n$, we can find $t>0$ so small that $t t_{i}\left|X_{i}-Y_{i}\right| \leqslant \omega, i=1, \ldots, n$. Thus, using (10) (since we can assume $t<1$ ), we have

$$
\begin{aligned}
d(x, y) & =\phi^{-1}\left[\sum_{i=1}^{n} \phi_{i}\left(\left|X_{i}-Y_{i}\right|\right)\right] \\
& =\left(\frac{1}{t}\right) \phi^{-1}\left[\sum_{i=1}^{n} \phi_{i}\left(t\left|X_{i}-Y_{i}\right|\right)\right] \\
& =\left(\frac{1}{t}\right) \phi^{-1}\left[\sum_{i=1}^{n} \phi\left(t t_{i}\left|X_{i}-Y_{i}\right|\right)\right] \\
& \left.=\left(\frac{1}{t}\right)\left[\sum_{i=1}^{n}\left(t t_{i}\left|X_{i}-Y_{i}\right|\right)^{r}\right]^{1 / r} \quad \text { (since } t t_{i}\left|X_{i}-Y_{i}\right| \leqslant \omega\right) \\
& =\left[\sum_{i=1}^{n}\left(t_{i}\left|X_{i}-Y_{i}\right|\right)^{r}\right]^{1 / r} \cdot
\end{aligned}
$$

Thus, $d$ is a power metric, for all points $x, y$ (and of course, by the uniqueness part of Theorem $1, \phi_{i}(\alpha)=k\left(t_{i} \alpha\right)^{r}$ in the large).

The restriction $1 \leqslant r<\infty$ is occasioned, not by Eqs. 10 or 12, but by the need to satisfy the triangle inequality; we refer the reader to standard discussions of the Minkowski inequality (e.g., Hardy et al., 1952, or any book on functional analysis).

\section{Psychological Dimensions and Metrics}

It has been shown in the previous sections that (A1)-(A6) yield additive difference measurement of dissimilarity between multidimensional objects. According to this model, dissimilarity judgments are described in terms of two sets of scales that apply to each one of the dimensions. The first set of scales, $f_{1}, \ldots, f_{n}$, applies directly to the physical input and describes its psychological counterpart along each one of the dimensions. We shall call them psychophysical functions. The second set of scales, $\phi_{1}, \ldots, \phi_{n}$, applies to the perceived component-wise differences along the dimensions, and describes their contributions to the overall dissimilarity between the stimuli. We shall call them similarity functions. In the additive difference model, dissimilarity 
judgments are decomposed into two independent processes: a "perceptual" process satisfying intradimensional subtractivity, and an "evaluative" process satisfying interdimensional additivity. Another version of the additive difference model, that is based on preferences rather than similarity judgments, was presented in Tversky (1969).

Although the general concept of a similarity function has not been formally introduced, related notions have been proposed in the analysis of similarity judgments. In discussing the results of a two-dimensional similarity experiment, Attneave (1950) writes:

a given change in the difference between two stimuli becomes less noticeable as the total difference between the stinuli is increased. It is lempting, therefore, to think of it as a sort of second order Weber-Fechner function. The abscissa is already related to the physical variable, if there are magnitudes, by a Fechner function or some suitable variant thereof; the present interpretation suggests that these first order psychological distances on the abscissa are pseudo-physical in their relationship to some higher perceptive level and they go through another Fechner transformation in being perceived [p. 552].

In the additive difference model, the stimuli are represented in a dimensionallyorganized space, but the dissimilarity ordering need not coincide with any metric. The assumption that an additive difference model is compatible with a proper metric imposes severe restrictions on the shape and the interrelations among the similarity functions, restrictions that may but need not be acceptable on empirical grounds. More specifically, if an additive difference model is compatible with a proper metric, all similarity functions must be essentially identical; moreover, they should all be superadditive.

In multidimensional scaling it is assumed that stimuli can be characterized by some (subjective) dimensions, and that the dissimilarity between them is compatible with a metric. It should be emphasized, however, that the dimensional and the metric assumptions are independent of each other, as each one of them can be satisfied without the other. The failure to distinguish between the metric and the dimensional assumptions hinders the explication of their empirical meaning and the clarification of their psychological justification. What, then, is the psychological meaning of metrics and dimensions?

The concept of a metric with additive segments has been analyzed in detail by Beals and Krantz (1967) (see also Beals et al., 1968), so we limit our discussion to the concept of a psychological dimension-a concept that has been used in the literature in several different senses.

One meaning of the term dimension is a variable that can be manipulated experimentally, such as the intensity of a tone, or the shape of a geometric figure. This use of the term dimension is neutral from a psychological standpoint since it refers to the way in which stimuli are specified or generated physically and not to the way in which 
they are perceived. Several physical dimensions (in the above sense) may combine into a single psychological dimension and vice versa.

A second meaning of the term dimension is a trait, or a variable, that cannot be observed or measured directly, but can be expressed in terms of other measurable traits or variables. The factor analytic definitions of pure neuroticism or of abstract reasoning ability are examples of dimensions that are defined as (linear) combinations of some measurable variables, such as various test scores. Hence, such definitions can be viewed as derived measurement. The scaling of these dimensions (though not their labeling) does not depend on any testable psychological assumptions, rather it attempts to express a large number of correlated variables in terms of a smaller number of uncorrelated ones.

The third, and by far the most interesting, meaning of the term dimension refers to the factors along which stimuli are perceived and structured. In speaking of hue, saturation, and brightness as dimensions of color space, or of potency as a dimension of semantic space, it is typically implied that these dimensions serve as organizing principles in the perception of colors or words. To accept such an interpretation, however, one has to demonstrate the role played by the alleged dimensions in the perception of objects. This can be done in two different ways.

One approach consists of collecting introspective data, of a direct or an indirect nature, about the manner in which objects are perceived. The second approach consists of constructing dimensional models and then testing whether any variable (specified physically or defined in terms of other variables) acts like a dimension as defined in the model. In this approach, therefore, a psychological dimension is defined in terms of its formal characteristics. Consequently, one can test which of several variables, if any, can be regarded as a dimension by studying its formal properties.

One necessary condition for defining a psychological dimension is the possibility of defining equivalence classes with respect to this dimension in a way that is independent of the levels of the other dimensions. Thus, if area, for example, is one of the psychological dimensions of geometric figures, then one would expect judgments concerning area to be independent of the shape of the figures. This may not be true when area is defined physically, but still be true when area is defined by a psychological rather than a physical operation. Indeed, this is the major difficulty in specifying psychological dimensions in physical units.

The possibility of defining equivalence classes in an independent fashion, however, does not exhaust the properties expected of a psychological dimension. Additional properties can be discovered by examining the models proposed for similarity data. The analysis of a large class of multidimensional similarity models shows that the dimensions of the space satisfy two major assumptions: (intradimensional) subtractivity and (interdimensional) additivity. These properties may, thus, be taken as defining properties for psychological dimensions. The axiomatic analysis of these properties reveals what are the testable ordinal assumptions that are necessary and/or sufficient 
for subtractivity and additivity to be satisfied. Hence, it provides methods for testing whether a given factor is a psychological dimension in the sense of satisfying subtractivity, and satisfying additivity with respect to some other dimensions.

The present approach is characterized by treating multidimensional scaling models as quantitative psychological theories rather than as methods for data reduction. It rests on the supposition that the axiomatic analysis of these models combined with the experimental investigation of their consequences will result in a deeper understanding of the metric and dimensional structures that underly similarity data.

\section{RFFERENCES}

AczÉL, J. Lectures on functional equations and their applications. New York: Academic Press, 1966. Atrneave, F. Dimensions of similarity. American Journal of Psychology, 1950, 63, 516-556.

Beals, R., AND Krantz, D. H. Metrics and geodesics induced by order relations. Mathematische Zeitschrift, 1967, 101, 285-298.

Beals, R., Krantz, D. H., ANo Tversky, A. Foundations of multidimensional scaling. Psychological Review, 1968, 75, 127-143.

Busemann, H. The geometry of geodesics. New York: Academic Press, 1955.

DeBREU, G. Representation of a preference ordering by a numerical function. In R. M. Thrall, C. H. Coombs, and R. L. Davis (Eds.), Decision processes. New York: Wiley, 1954. Pp. 159-165.

Debreu, G. Topological methods in cardinal utility theory. In K. J. Arrow, S. Karlin, and P. Suppes (Eds.), Mathematical methods in the social sciences. Stanford: Stanford Univer. Press, 1960. Pp. 16-26.

HARDY, G. H., Littlewood, J. E., AND POLYA, G. Inequalities. London/New York: Cambridge Univer. Press, 1952.

KreIn, M., AND Milman, D. On the extreme points of regularly convex sets. Studia Mathematica, 1940, 9, 133-138.

KRANTZ, D. H. A survey of measurement theory. In G. B. Dantzig and A. F. Veinott, Jr. (Eds.), Mathematics of the decision sciences, Part 2. (Lectures in Applied Mathematies, Vol. 12). Providence, R. I.: American Mathematical Society, 1968. Pp. 314-350.

LucE, R. D. Two extensions of conjoint measurement. Journal of Mathematical Psychology, $1966,3,348370$.

LuCE, R. D., AND Tukey, J. W. Simultaneous conjoint measurement: A new type of fundamental measurement. Journal of Mathematical Psychology, 1964, 1, 1-27.

SUPPES, P., AND WINET, M. An axiomatization of utility based on the notion of utility differences. Management Science, 1955, 1, 259-270.

TVERSky, A. Intransitivity of preferences. Psychological Review, 1969, 76, 31-48.

Tversky, A., AND Krantz, D. H. Similarity of schematic faces: A test of interdimensional additivity. Perception \& Psychophysics, 1969, 5, 124-128.

Received: July 3, 1969 